\title{
Lipid-based nanocarriers to enhance skin permeation and antioxidant activity of Centella asiatica extract
}

\author{
Boonnada Pamornpathomkul ${ }^{1}$, Worranan Rangsimawong ${ }^{1}$, Theerasak Rojanarata ${ }^{1}$, Praneet Opanasopit ${ }^{1}$ \\ Chuleerath Chaiyodsilp ${ }^{2}$ and Tanasait Ngawhirunpat ${ }^{1, *}$ \\ ${ }^{1}$ Pharmaceutical Development of Green Innovations Group (PDGIG), Faculty of Pharmacy, Silpakorn University, \\ Nakhon Pathom, Thailand. \\ ${ }^{2}$ Thai Herb for Hair Co., Ltd. Nakhon Pathom, Thailand
}

\begin{abstract}
The purpose of this study was to evaluate the use of different formulations, including solution, gel, liposome and niosome for in vitro skin permeation and antioxidant activity of Centella asiatica (CA) extract. The liposomes and niosomes loaded with CA were characterized to observe the physicochemical properties i.e., particle size, zeta potential, percentage of entrapment efficiency (\%EE) and percentage of loading efficiency (\%LE). In vitro skin permeation studies revealed that liposome formulations had a superior enhancing effect on skin permeation compared to niosome, gel and solution formulation. Upon applied niosome formulations for the delivery of CA extract at 24 hours (h), the antioxidant activity was higher than liposome, gel and solution formulation, as evidenced by the increased in percent inhibition using 2,2-diphenyl-1-picrylhydrazyl (DPPH) assay. However, there was no significant difference in antioxidant activity between niosome and liposome formulations. Accordingly, both the liposome and noisome formulations are promising approaches for transdermal delivery of CA extract for promoting successful antioxidant activity.
\end{abstract}

\section{Introduction}

Centella asiatica (CA) is a tropical medicinal plant from the Apiaceae family native to Southeast Asian countries. It is commonly known as "bua-bok" in Thailand. It has been widely used as a traditional herbal medicine due to biological activities from asiaticoside as a major constituent of CA, including being an antioxidant [1], anti-aging, antipsoriatic [2] and anti-inflammatory [3]. $\mathrm{CA}$ is the chief herb for treating hair loss and promoting hair re-growth, which strengthens the hair blood vessels and expands hair follicles due to the entry of more nutrients and oxygen. In addition, antioxidant agents can interrupt radical chain processes, protect against oxidative damage and help to repair hair systems [4].

It is challenging to deliver molecules into the skin because of the foremost barrier from the stratum corneum of skin [5]. Therefore, improved delivery strategies are necessary to solve this issue. Lipid-based nanocarriers are attractive formulations for topical and transdermal drug delivery. Among the lipid-based nanocarriers systems, liposomes have been widely investigated as potentially efficacious lipid nanocarriers. Liposomes are small, sphere-shaped vesicles. They can be created from cholesterol and natural nontoxic phospholipids resulting in being consisted of amphipathic phospholipids arranged in one or more phospholipid bilayer(s). Hydrophilic drugs can be entrapped within the inner aqueous core and hydrophobic drugs can be intercalated in the lipid bilayers. Due to the small size vesicle, hydrophobic and hydrophilic character, liposomes have the ability to interact with lipids in skin and decreased systemic absorption [6].

Niosomes are also widely used as lipid-based nanocarriers for drug delivery. Although liposomes can encapsulate various types of drugs and are advantageous, their formulation is expensive and there is a limited shelf life. Niosomes have the functional and physicochemical properties similar to a liposome vesicle. However, niosomes differ from liposomes in their chemical structure resulting in greater stability than liposomes [7]. Niosomes are created from cholesterol and non-ionic surfactants resulting in bilayer structures being formed. They are composed of amphiphilic molecules surrounded by an aqueous compartment that contain both hydrophobic and hydrophilic groups [8]. Niosome nanocarriers have been used as potential transdermal drug delivery systems due to enhanced drug permeation and localized depots for sustained drug release [9].

In this study, CA extract was selected as our model compound because it is a hydrophilic compound with many biological activities, especially, antioxidant activity and promotion of hair growth. Physicochemical characterizations were investigated to compare the different lipid nanocarriers. Moreover, the effect of different formulations, i.e., CA solution, CA gel, CA liposome and CA niosome on skin permeation and antioxidant activity were investigated.

\section{Materials and methods}

\subsection{Materials}

\footnotetext{
* Corresponding author: ngawhirunpat $\mathrm{t} @$ su.ac.th
} 
Egg phosphatidylcholine (PC) was purchased from Lipoid GmbH (Ludwigshafen, Germany). Cholesterol was obtained from Carlo Erba Reagent (Ronado, Italy). Span 20, DPPH and d-limonene were purchased from Sigma-Aldrich (MO, U.S.A.). All chemicals used were of analytical reagent grade.

\subsection{Preparation of CA extract}

Fresh leaves of $\mathrm{CA}$ were dried at $50^{\circ} \mathrm{C}$, powdered and sifted. Powder was refluxed with $36 \%$ ethanol at $60^{\circ} \mathrm{C}$ for $1 \mathrm{~h}$. The extract was then filtered, mixed with $80 \%$ propylene glycol and stored at $4{ }^{\circ} \mathrm{C}[10]$. To control the concentration of extract, high-performance liquid chromatography (HPLC) was used to control the initial concentration of CA extract $(4 \% \mathrm{w} / \mathrm{v})$.

\subsection{Preparation of formulations}

\subsubsection{CA gel preparation}

The gel formulation was prepared by dispersing $4 \%$ of Carbopol $^{\circledR} 940$ polymer into the deionized water. Then, $4 \% \mathrm{w} / \mathrm{v}$ of $\mathrm{CA}$ extract was added and mixed until homogeneous. Triethanolamine was then added with gentle sweeping agitation.

\subsubsection{CA liposome preparation}

Liposome formulations were prepared by thin film hydration method. Briefly, $250 \mu \mathrm{l}$ of PC $(200 \mathrm{mM})$ and $500 \mu$ l of cholesterol $(20 \mathrm{mM})$ were dissolved with $2: 1$ $\mathrm{v} / \mathrm{v}$ of chloroform/methanol. The solvent was then evaporated using stream of nitrogen. The thin film was placed in a desiccator until completely dry $(6 \mathrm{~h})$. The $4 \%$ CA extract was dissolved in phosphate-buffered saline (PBS; pH 7.4) and added to the lipid film to give hydrated liposome vesicles. The dispersion was mixed with Tween 20 and d-limonene. The formulation was sonicated for $30 \mathrm{~min}$ to reduce the size of the liposomes. Remaining materials were removed by centrifuge at $15,000 \mathrm{rpm}$ for $15 \mathrm{~min}$. The supernatant was collected.

\subsubsection{CA niosome preparation}

The niosome formulation was formulated similarly with the method used for the liposomes. Briefly, $125 \mu \mathrm{l}$ of Span $20(200 \mathrm{mM})$ and $125 \mu \mathrm{l}$ of cholesterol $(200 \mathrm{mM})$ were dissolved with chloroform/ethanol (1:1). The solvent was evaporated with a stream of nitrogen and then placed in a desiccator for $6 \mathrm{~h}$. The $4 \% \mathrm{CA}$ extract was dissolved in PBS ( $\mathrm{pH} 7.4) 5 \mathrm{ml}$ and added to the lipid film to give hydrated niosome. The dispersion was mixed with Tween 20 and d-limonene. The formulation was sonicated for $30 \mathrm{~min}$. Remaining materials were also removed. The supernatant was collected.

\subsection{Characterization of nanocarrier formulations}

\subsubsection{Particle size and zeta potential analysis}

The particle size and surface charge of the nanocarrier formulations were determined at $25^{\circ} \mathrm{C}$ by photon correlation spectroscopy using a Zetasizer Nano ZS (Malvern Instrument, Worcestershire, U.K.).

\subsubsection{Percentage of entrapment efficiency (\%EE) and loading efficiency (\%LE)}

Each lipid nanocarrier formulation $(0.5 \mathrm{~mL})$ was placed in a Microcon YM-3 ultrafiltration (Minipore, Billerica, U.S.A.) and centrifuged at $10000 \times \mathrm{g}$ at $4^{\circ} \mathrm{C}$ for $60 \mathrm{~min}$. The filtrate was discarded, and $0.25 \mathrm{~mL}$ of PBS was then added to the retentate before further centrifugation at $4^{\circ} \mathrm{C}$ at $10000 \times \mathrm{g}$ for $40 \mathrm{~min}$ [11]. The collected CAloaded nanocarriers in the retentate were then disrupted with $0.1 \% \mathrm{w} / \mathrm{v}$ TritonX-100 and centrifuged at $4^{\circ} \mathrm{C}$ at $10000 \times \mathrm{g}$ for $10 \mathrm{~min}$. The CA contents of the supernatants were determined using HPLC. The drug $\% \mathrm{EE}$ and $\% \mathrm{LE}$ were calculated with equations 1 and 2 :

$$
\begin{gathered}
\% \mathrm{EE}=\left(C / C_{i}\right) \times 100 \\
\% \mathrm{LE}=(C / \text { Lipid composition }) \times 100
\end{gathered}
$$

where $C$ is the concentration of CA extract in the formulation, and $C_{i}$ is the initial concentration of $\mathrm{CA}$ extract.

\subsection{In vitro skin permeation studies}

A Strat-MTM membrane was used as a human skin model because it has multiple layers with varied diffusivity as it correlates more closely to human skin than animal skin models. The Strat-M membranes were mounted between the donor and the receptor compartments of Franz diffusion cells with the epidermis side facing up towards the donor compartment, and the apparatus was affixed using clamps. The receptor compartment was filled with PBS and continuously stirred at $400 \mathrm{rpm}$. To evaluate the effect of different formulations, including CA solution, gel, liposome and niosome, the concentration of CA extract placed into the donor compartment was $4 \% \mathrm{w} / \mathrm{v}$. Two milliliters of different CA formulations were placed onto the skin. The top of the donor compartment was then covered with Parafilm ${ }^{\circledR}$ for occlusion. The temperature was maintained at $32 \pm 1$ ${ }^{\circ} \mathrm{C}$. To investigate the cumulative permeation profiles, a $500 \mu \mathrm{l}$ aliquot of receptor was withdrawn at the time intervals of 2, 4, 6, 8 and $24 \mathrm{~h}$ and replaced with equal volumes of fresh PBS. Each sample was divided for measuring the concentration of asiaticoside using HPLC and antioxidant activity by DPPH assay.

After the concentration of asiaticoside was measured using HPLC, the cumulative amount of asiaticoside permeated into the skin samples was plotted against time, and the steady-state flux was determined as the slope of the linear portion of the plot. The lag time was obtained by extrapolating the linear portion of the 
penetration profile to the abscissa. The enhancement ratio (ER) was calculated with equation 3 :

$$
\mathrm{ER}=\frac{\text { Flux of nanocarrier formulations }}{\text { Flux of } 4 \% \text { CA solution }}
$$

\subsection{Quantitative analysis of asiaticoside}

The amount of asiaticoside permeated through Strat-M ${ }^{\circledR}$ membrane was quantized using HPLC 1260 infinity Agilent Technologies ${ }^{\circledR}$ (California, U.S.A.) with a UV detector. Chromatographic separation was performed with a Zobrax Eclipse XDB-C18 ${ }^{\circledR}$ column $(4.6 \times 250 \mathrm{~mm}$ I.D.; particle size: $5 \mu \mathrm{m}$ ) (Agilent Technologies, California, U.S.A.). Mobile phase was a gradient of acetonitrile/water as shown in Table 1, a flow rate of 1 $\mathrm{ml} / \mathrm{min}$ and detection at $206 \mathrm{~nm}$.

Table 1. Gradient conditions for HPLC

\begin{tabular}{|c|c|c|}
\hline Time (min) & $\begin{array}{c}\text { Pump A, } \\
\text { water (\%) }\end{array}$ & $\begin{array}{c}\text { Pump B, } \\
\text { acetronitrile (\%) }\end{array}$ \\
\hline 0 & 80 & 20 \\
\hline 15 & 65 & 35 \\
\hline 30 & 35 & 65 \\
\hline 35 & 20 & 80 \\
\hline 40 & 20 & 80 \\
\hline
\end{tabular}

\subsection{Antioxidant activity}

The DPPH assay method was used in this study. This method was based on the reduction of DPPH, a stable free radical. The free radical DPPH with an odd electron gives a maximum absorption at $517 \mathrm{~nm}$ (purple color). Upon reduction, the solution color fades; the reaction progress is monitored by a spectrophotometer. Onehundred microliters of samples were added into each well of 96-well plates. A $100 \mu 1$ of DPPH solution (200 $\mu \mathrm{M}$ ) was then added, mixed and left at $25^{\circ} \mathrm{C}$ for $30 \mathrm{~min}$. The absorbance was then measured at $550 \mathrm{~nm}$ using a microplate reader. The results of the assay were expressed as \%inhibition (equation 4).

$$
\% \text { inhibition }=\left(1-\mathrm{A}_{\text {sample }} / \mathrm{A}_{\text {control }}\right) \times 100
$$

Where $\mathrm{A}_{\text {sample }}$ and $\mathrm{A}_{\text {control }}$ are the absorbance in the presence of the different formulations and control, respectively.

\subsection{Data analysis}

All data were statistically analyzed by one-way analysis of variance (ANOVA). In all cases, a value $p<0.05$ was considered statistically significant.

\section{Results and Discussions}

\subsection{The physiochemical characterization of lipid formulations}

\subsubsection{Particle size and zeta potential}

The physiochemical characteristics of the different lipid formulations are shown in Table 2. The lipid nanocarrier formulations showed the nanoscale range of particle size with a negative charge. The average particle size of the liposome formulation was smaller than the niosome formulation. Moreover, the liposome formulation showed a narrow size distribution (polydispersity index; $\mathrm{PDI}<0.3$ ), while the niosome formulation has large variability in the particle size. An increase in the particle size of niosomes might be due to the interaction of drug with surfactant head groups of niosomes resulting in increasing the charge and repulsion force of the surfactant bilayers [12].

\subsection{2 \%Entrapment efficiency and \%loading efficiency}

As shown in table 2, the \%EE of the liposome formulation was higher than that of the niosome formulation, suggesting that the niosome structure was more rigid and decreased drug entrapment. The drug entrapment efficiency of niosomes was lower than liposomes. An increase in cholesterol content of the niosome formulation resulted in a decrease in the fluidity of the niosome bilayers but an increase in the drug loading efficiency. Moreover, the thickness of the niosome membrane structure and solubility of the drug in water affected the drug loading efficiency [13]. In case of the liposome formulation, the drug loading efficiency depends on drug-lipid interactions, the solubility of the drug in the liposome membrane and the ability of liposomes to encapsulate aqueous drugs during vesicle formation $[6,14]$

Table 2. Characterization parameters of the lipid nanocarrier formulations

\begin{tabular}{|c|c|c|}
\hline Parameters & CA liposome & CA niosome \\
\hline Particle size (nm) & $42.33 \pm 0.47$ & $55.73 \pm 2.52$ \\
\hline PDI & $0.28 \pm 0.03$ & $0.43 \pm 0.01$ \\
\hline Zeta potential (mV) & $-13.30 \pm 0.64$ & $-3.50 \pm 0.33$ \\
\hline \% EE & $94.31 \pm 3.84$ & $72.55 \pm 2.74$ \\
\hline \%LE & $16.93 \pm 0.69$ & $28.94 \pm 1.26$ \\
\hline
\end{tabular}

\subsection{In vitro skin permeation studies}

The in vitro skin permeation profiles of asiaticoside from the different formulations are illustrated in Fig. 1A and 1B. The permeation parameters of asiaticoside from the different formulations are shown in Table 3. The cumulative amount of asiaticoside at $24 \mathrm{~h}$ after applying various formulations could be ranked as follows: CA liposome $>$ CA niosome $>$ CA gel $>$ CA solution. The results showed that the $\mathrm{CA}$ liposome formulation was significantly increased in the skin permeation of asiaticoside when compared with other formulations. Although CA niosome formulation slightly enhanced the skin permeation of asiaticoside in comparison with the 
gel formulation, there was no significant difference in cumulative amounts of asiaticoside at $24 \mathrm{~h}$. The most enhancing effect of liposome formulation might be caused from the lipid composition of liposome structures, as it was similar to the epidermis layer of the skin which enables them to penetrate the epidermal barrier to a greater extent compared to other formulations [15]. Moreover, the liposome formulation forms a barrier around their drugs, which is resistant to oxidation and degradation [6]. Compared with the liposome formulation, the skin permeation of the niosome formulation was significantly lower. The particle size of the liposome formulation was smaller and the $\% \mathrm{EE}$ of the liposome formulation was higher than the niosome formulation. In addition, CF loaded into the liposomes with higher \%EE could present higher skin permeation than those with lower \%EE [16]. Therefore, it could be assumed that the particle size and \%EE substantially affect the transdermal drug delivery of lipid-based nanocarriers.

A
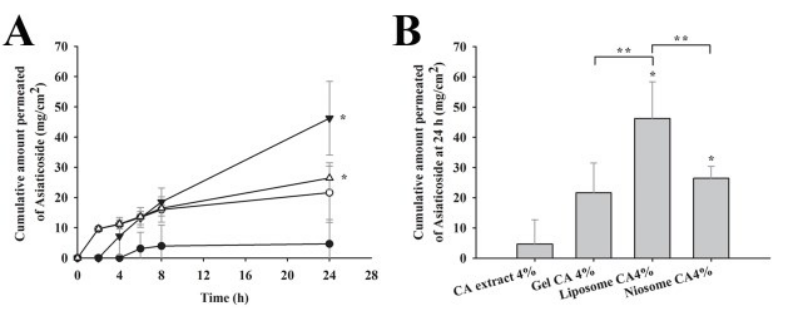

Fig. 1. In vitro permeation profiles of asiaticoside (A) The cumulative amount profiles of asiaticoside in (O) CA solution,

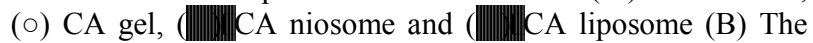
cumulative amount at $24 \mathrm{~h}$ of all groups. * indicates significant difference compared to CA solution $(p<0.05)$. **indicates significant difference between two groups $(p<0.05)$.

Table 3. The permeation parameters of asiaticoside from different formulations

\begin{tabular}{|c|c|c|c|c|}
\hline $\begin{array}{l}\text { Formu- } \\
\text { lations }\end{array}$ & $\begin{array}{c}\text { Flux } \\
\left(\mathrm{mg} / \mathrm{cm}^{2} / \mathrm{h}\right)\end{array}$ & ER & $\begin{array}{l}\text { Lag time } \\
\text { (h) }\end{array}$ & $\begin{array}{c}K_{p}\left(\times 10^{-3}\right) \\
(\mathrm{cm} / \mathrm{h})\end{array}$ \\
\hline solution & $0.2036 \pm 0.35$ & - & $0.46 \pm 0.80$ & $0.10 \pm 0.18$ \\
\hline gel & $0.7084 \pm 0.41$ & 3.48 & $6.77 \pm 0.55$ & $0.36 \pm 0.21$ \\
\hline liposome & $1.9846 \pm 0.49^{*, * *}$ & 9.56 & $0.05 \pm 0.08$ & $1.01 \pm 0.25^{*, * *}$ \\
\hline niosome & $0.9222 \pm 0.17^{*, * *}$ & 4.53 & $6.14 \pm 0.45$ & $0.47 \pm 0.08^{*, * *}$ \\
\hline
\end{tabular}

\subsection{Antioxidant activity}

The niosome formulation had a superior antioxidant activity after $24 \mathrm{~h}$ of being topically applied compared to the liposome, solution and gel formulation, respectively (Fig.2). Antioxidant levels were found to increase significantly in both the liposome and niosome formulations after $24 \mathrm{~h}$ of being topically applied. Although the niosome formulation slightly improved antioxidant activity in comparison with liposome formulation, there was no significant difference between percentage inhibitions at $24 \mathrm{~h}$. It can be concluded that both the liposomes and niosomes are very suitable formulations for transdermal delivery of CA extract.

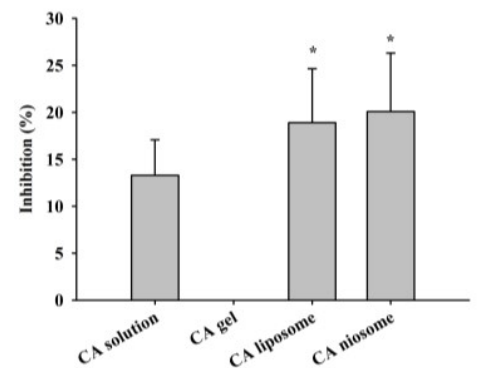

Fig. 2. Antioxidant activity of DPPH assay of different CA formulations

\section{Conclusion}

The purpose of this study was to evaluate the different lipid formulations with CA extract on skin permeation and antioxidant activity. The particle size of lipid-based nanocarrier formulations was in the nanoscale range. Both liposomes and niosomes revealed a high cumulative amount at $24 \mathrm{~h}$ and antioxidant activity of CA extract. The ability of liposomes and niosomes to enhance drug delivery and promoting antioxidant activity promotes the two lipid-based nanocarriers to be useful for topical formulations for CA extract.

\section{Acknowledment}

We gratefully acknowledge the financial support provided by the National Innovation Agency, Thailand.

\section{References}

1. M. Bajpai, A. Pande, S.K. Tewari, D. Prakash, Int J Food Sci Nutr, 56 (2005).

2. J.H. Sampson, A. Raman, G. Karlsen, H. Navsaria, I.M. Leigh, Phytomedicine, 8 (2001).

3. M. George, L. Joseph, Ramaswamy, African Journal of Traditional, Complementary, and Alternative Medicines, 6 (2009).

4. M.P. Lupo, Clin Dermatol, 19 (2001).

5. O.G. Jepps, Y. Dancik, Y.G. Anissimov, M.S. Roberts, Adv Drug Deliv Rev, 65 (2013).

6. A. Akbarzadeh, R. Rezaei-Sadabady, S. Davaran, S.W. Joo, N. Zarghami, Y. Hanifehpour, M. Samiei, M. Kouhi, K. NejatiKoshki, Nanoscale Res Lett, 8 (2013).

7. N.B. Mahale, P.D. Thakkar, R.G. Mali, D.R. Walunj, S.R. Chaudhari, Advances in Colloid and Interface Science, 183184 (2012)

8. I.F. Uchegbu, A.T. Florence, Advances in Colloid and Interface Science, $\mathbf{5 8}$ (1995).

9. R. Muzzalupo, L. Tavano, Research and Reports in Transdermal Drug Deliver, 4 (2015).

10. P. Hashim, H. Sidek, M.H. Helan, A. Sabery, U.D. Palanisamy, M. Ilham, Molecules, 16 (2011).

11. W. Rangsimawong, P. Opanasopit, T. Rojanarata, S. Duangiit, T. Ngawhirunpat, Biol Pharm Bull, 39 (2016).

12. K.M. Kazi, A.S. Mandal, N. Biswas, A. Guha, S. Chatterjee, M. Behera, K. Kuotsu, Journal of Advanced Pharmaceutical Technology \& Research, 1 (2010).

13. B. Shi, C. Fang, Y. Pei, J Pharm Sci, 95 (2006).

14. S. Hua, Frontiers in Pharmacology, 6 (2015).

15. J. Dua, A. Rana, A. Bhandari, Int J Pharm Stud Res, 3 (2012).

16. D.D. Verma, S. Verma, G. Blume, A. Fahr, Int J Pharm, 258 (2003). 